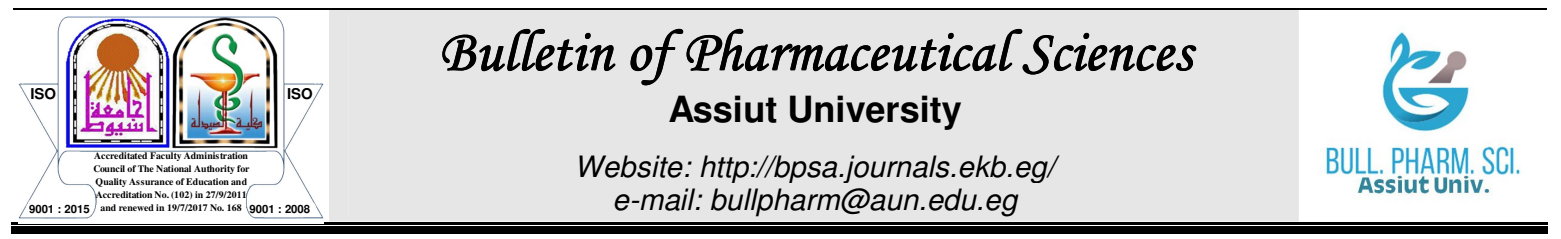

\title{
THE GALA PEPTIDE: MULTIPLE ROLES IN DRUG AND GENE DELIVERY
}

\author{
Ikramy A. Khalil ${ }^{1, *}$ and Hideyoshi Harashima ${ }^{2}$ \\ ${ }^{1}$ Department of Pharmaceutics, Faculty of Pharmacy, Assiut University, Assiut 71526, Egypt \\ ${ }^{2}$ Faculty of Pharmaceutical Sciences, Hokkaido University, Kita-12, Nishi-6, Kita-ku, Sapporo \\ 060-0812, Japan
}

\begin{abstract}
Therapeutic nucleic acids must be formulated in delivery systems that permit them to be efficiently targeted to specific tissues within the body and to overcome the endosomal barrier to deliver these molecules to their intracellular target sites. GALA is a synthetic amphipathic 30 amino acids peptide that performs both functions. It has the ability to target the lung endothelium and induce membrane fusion, which facilitates endosomal escape of therapeutic nucleic acids. In this review, we introduce the GALA peptide and discuss its multiple roles in drug and gene delivery. We first describe the peptide and its ability to induce membrane fusion and pore-formation. We next focus on applications of this peptide in gene delivery in-vitro and in-vivo. We also discuss the synergistic combination between GALA and oligoargnine peptides and their applicability to active tumor targeting. The GALA peptide introduced in this review has great promise as an efficient tool for gene delivery.
\end{abstract}

\section{INTRODUCTION}

The use of genes or nucleic acids such as DNA or RNA as novel therapeutics is a recent trend in nanomedicine and represents a highly promising strategy for the treatment of many currently incurable diseases ${ }^{1}$. Nucleic acids do not function alone as drugs, but rather need to be formulated in specially designed delivery systems to be effective ${ }^{2}$. In fact, nucleic acid delivery is more sophisticated and more challenging compared to the delivery of conventional, low molecular weight drugs. Nucleic acids are easily degraded inside the body and their target sites are always located inside the cells in the cytosol or nucleus. They do not generally penetrate the cell wall because they are highly charged, relatively high molecular weight molecules. Therefore, they must be formulated in appropriate delivery systems, which provide adequate protection and permit these molecules to be efficiently delivered to specific tissues within the body. Nevertheless, the delivery system must overcome different extracellular and intracellular obstacles in order to reach their target sites inside the cell. Although viral vectors are more efficient for gene delivery compared to non-viral vectors, their use is accompanied by several risks including toxicity, immunogenicity and possibility of infections ${ }^{3}$. Non-viral vectors are generally safer and more convenient for large scale production, although their efficiency is relatively low ${ }^{4 \& 5}$.

To increase the efficiency of non-viral gene delivery, new biomaterials need to be synthesized to overcome the different obstacles. In addition, innovative gene delivery systems need to be designed to integrate different functional biomaterials in a manner that allow these materials to operate at their full potential at the optimal time and place $^{6}$. An ideal gene delivery system must efficiently target specific tissues in the body and must be capable of delivering therapeutic nucleic acids to their target sites in the cytosol or the nucleus. This is a challenging task because systemically injected nucleic acids are rapidly opsonized in the blood and delivered to the

Received in 4/2/2020 \& Accepted in 29/2/2020 
liver for degradation ${ }^{7}$. In addition, they are internalized into cells through endocytosis and must escape from the endosomal compartment in order to reach the cytosol ${ }^{8}$. Therefore, a biomaterial that can perform both functions; i.e. tissue targeting and endosomal escape, would be an excellent tool for gene delivery. The GALA peptide can perform this double function efficiently. This synthetic 30 amino acid amphipathic peptide shows strong fusogenic activity at an acidic $\mathrm{pH}$ as in late endosomes, which facilitates the endosomal escape of therapeutic genes ${ }^{9}$. Furthermore, our group recently discovered that this peptide has a strong targeting ability to the lung endothelium ${ }^{10}$. In addition, we have recently shown that this peptide functions synergistically in conjunction with certain cell penetrating peptides (CPPs) through dramatically improving cellular uptake and endosomal escape via enhancing the interactions between different lipid membranes ${ }^{11}$. Therefore, the GALA peptide plays multiple roles in gene delivery and can be regarded as a strong candidate biomaterial for developing efficient non-viral gene delivery systems.

In this review, we introduce the GALA peptide and discuss its multiple roles in drug and gene delivery. We first describe the peptide and summarize its properties and its ability to induce fusion and pore formation. We next focus on applications of the GALA peptide in gene delivery in-vitro and in-vivo. We provide various experimental evidence which support the role of this peptide in enhancing endosomal escape and targeting the lung endothelium. We also discuss the synergistic combination between the GALA peptide and selected CPPs and its applicability in active tumor targeting. The uptake mechanism of GALA-modified nanoparticles (NPs) and their ability to reach alveolar epithelial cells in the lung through transcytosis will be discussed. We believe the GALA peptide introduced in this review carries great promise as an efficient tool for gene delivery.

\section{The GALA peptide}

\section{1- Structure and binding to nucleic acids}

GALA was originally synthesized by Szoka Jr. et al. to mimic the sequence of viral fusogenic peptides to study the perturbation of lipid bilayers and fusion between lipid membranes ${ }^{12}$. However, it rapidly became an important tool for enhancing the endosomal escape in gene delivery due to its strong ability to induce fusion between membranes at an acidic $\mathrm{pH}$. It is generally described in the area of drug delivery as "a fusogenic peptide" or "a pore-forming peptide". GALA is a synthetic 30 amino acid peptide whose sequence is (WEAALAEALAEALAEHLAEALAEALEAL AA $)^{9}$. It is generally regarded as being composed as repeating units (glutamic acidalanine-leucine-alanine, or EALA). The peptide exists as a random coil in neutral $\mathrm{pH}$ and converts to an $\alpha$-helix conformation in acidic $\mathrm{pH}^{9}$. The length of the peptide was chosen to confirm the stability of $\alpha$-helix at acidic $\mathrm{pH}$ and to ensure that the lipid bilayer can be crossed. The peptide is described as an amphipathic peptide where leucine (Leu) residues confer sufficient hydrophobicity and glutamic acid (Glu) residues confer hydrophilicity and negative charge. Glu residues are protonated at acidic $\mathrm{pH}$ and this adds to the hydrophobicity of the peptide and facilitates its binding with different lipid membranes.

In neutral $\mathrm{pH}$, electrostatic repulsion due to the several negative charges of the Glu residues allows the peptide to maintain a random coil conformation. Upon acidification of the medium ( $\mathrm{pH} \mathrm{5),} \mathrm{the} \mathrm{neutralization} \mathrm{of} \mathrm{the}$ carboxylic acids groups in Glu residues decreases the extent of electrostatic repulsions and promotes the formation of an $\alpha$-helix structure (Fig. 1). The amino acid sequence was carefully selected so that the $\mathrm{pH}$-sensitive Glu residues exist on one side of the $\alpha$-helix while the hydrophobic Leu residues are located on the other side and alanine (Ala) residues act as a spacer between the two sides. Segregation of amino acids on the two sides of the helix confers the amphipathic behavior.

Since GALA has a negative charge at neutral $\mathrm{pH}$, it has no ability to bind to negatively charged nucleic acids, which definitely decreases its potential for applications in gene delivery. To be used in gene delivery, GALA was mixed with nucleic acid complexes where it binds electrostatically to cationic components of these complexes $^{13 \& 14}$. Alternatively, GALA was chemically conjugated to different cationic 
lipids or polymers, which were then used to condense nucleic acids into stable NPs ${ }^{15}$. Our group postulated the synthesis of cholesterolGALA (Chol-GALA) as a derivative of GALA, which can be easily incorporated into lipid nanoparticles (LNPs) encapsulating different nucleic acids ${ }^{16}$. The cholesterol moiety acts as an anchor to the lipid layer of LNPs leaving the 30 amino acid GALA peptide freely exposed on the surface (Fig. 1). The use of Chol-GALA was essential for enhancing endosomal escape since unmodified GALA had no effect ${ }^{16}$. As an alternative strategy, a positively charged peptide, KALA, was synthesized by replacing the Glu residues in GALA with positively charged lysine (Lys) residues ${ }^{17}$. KALA has a sufficient positive charge to allow it to directly bind to nucleic acids and keep the other characteristics necessary for membrane fusion.
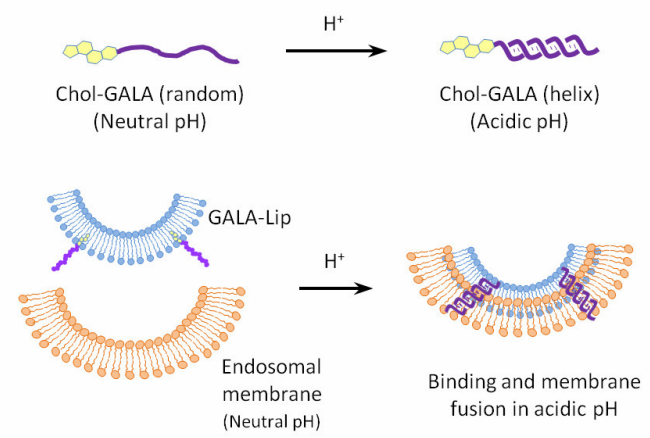

Fig. 1: A schematic representation of Chol-GALA conformation and the interaction between GALA-liposmes (GALA-Lip) and the endosomal membrane. GALA is conjugated with cholesterol to act as an anchor to the lipid of the liposomes. After endocytosis, GALA exists as a random coil at neural $\mathrm{pH}$ and a low interaction occurs between the GALA-Lip and the negatively charged endosomal membrane. Maturation of endosomes is associated with a decreased $\mathrm{pH}$, which causes the formation of a hydrophobic $\alpha$-helix that binds to the endosomal membrane. Subsequent membrane fusion and pore formation allow the contents of the liposomes to be released into the cytosol.

\section{2- Properties}

At $\mathrm{pH}$ 7, GALA in aqueous solutions exists mostly as a random coil. At this $\mathrm{pH}, 19 \%$ of the amino acids adopt a helix conformation while $20 \%$ are organized in $\beta$-sheets as judged by infrared attenuated total reflection spectroscopy $(\mathrm{ATR})^{18}$. When the $\mathrm{pH}$ is decreased to 5 , the helix conformation increased to $69 \%$ and the $\beta$-sheets increased to $28 \%$, and this change was correlated with the protonation of Glu residues. The helix conformation was stable at temperatures up to $40^{\circ} \mathrm{C}$ and increased with increasing salt concentration $^{12}$. At neutral $\mathrm{pH}$, the presence of divalent cations such as $\mathrm{Ca}^{++}$and $\mathrm{Zn}^{++}$ increased the helix content through neutralization of the Glu residues.

\section{3- Interaction with lipid membranes}

When mixed with large unilamellar vesicles (LUVs) composed of egg phosphatidylcholine (EPC), GALA could strongly bind to LUVs, but only at an acidic $\mathrm{pH}^{19}$. Studying the secondary structure of the peptide after binding to lipid vesicles revealed that the peptide entirely exists as an $\alpha$-helix, indicating that binding to lipid vesicles stabilizes the helix conformation. This binding is lost when the $\mathrm{pH}$ is raised to 7 , indicating that the binding is reversible and highly dependent on $\mathrm{pH}$. A lipid mixing assay was used to examine the fusion ability of GALA peptide. Two types of small unilamellar vesicles (SUVs) made of POPC were mixed in the presence of the peptide and the degree of lipid mixing was monitored using fluorescence resonance energy transfer (FRET) analysis ${ }^{20}$. The GALA peptide induced lipid mixing only at an acidic $\mathrm{pH}$ and this mixing was peptide concentration dependent. Dynamic light scattering showed that the diameter of the lipid vesicles increased upon acidification, indicating aggregation or fusion of smaller vesicles. The increased size was not affected by increasing the $\mathrm{pH}$ to 7 , which indicates that the increased size is due to fusion between lipids and not aggregation. In addition to membrane fusion, GALA induced pore formation in LUVs made of EPC in a pH-dependent manner. Using a specific leakage assay, GALA was shown to release the contents of EPC LUVs at $\mathrm{pH} 5^{21}$. The leakage started immediately after the addition of the peptide and rapidly reached $\sim 60 \%$ within $1 \mathrm{~min}$. The leakage then increased to $\sim 80 \%$ within $10 \mathrm{~min}$. The extent of leakage was increased with increasing peptide concentration. 
Based on these results, a model was proposed to describe the interaction of GALA with different lipid vesicles at acidic $\mathrm{pH}^{21}$. The peptide exists in a helix conformation in acidic $\mathrm{pH}$ and can bind to the lipid vesicles and induce lipid mixing. The peptide then aggregates and aligns so that the helix axis is perpendicular to the bilayer surface. This aggregation leads to the formation of aqueous pores, which allow the leakage of vesicle contents if they are large enough. The model assumed that 8-12 monomer helix units are assembled to form a pore in the lipid vesicle ${ }^{21}$. Applying this model to the gene delivery process, the gene carrying LNPs are generally internalized into cells through endocytosis. In endosomes, the $\mathrm{pH}$ continues to decrease which causes the GALA to change to the helix conformation that can bind to the inner side of the endosomal vesicles (Fig. 1). The peptide causes lipid mixing between the endosomal membrane and the lipid membrane of LNPs with destabilization and possible flip flop of the endosomal membrane leading to the release of the nucleic acids to the cytosol. Alternatively, the peptide aggregates in the endosomal membrane and forms aqueous pores which allow the content of the endosomes to be released to the cytosol. In summary, the presence of the GALA peptide strongly enhances endosomal escape and this is reflected as a dramatic improvement of gene expression efficiency as will be discussed later.

The role of GALA in gene delivery: Enhancing endosomal escape

\section{1- In-vitro gene delivery}

Due to its membrane disrupting ability, GALA is frequently used as a tool for enhancing the endosomal escape of nucleic acids. In early research, GALA was added to DNA complexes formed with poly-l-lysine (PLL) and transferrin (Tf $)^{13}$. GALA binds electrostatically to these positively charged complexes and significantly improved the gene expression in cultured cells. The increased gene expression was correlated with improved endosomal escape as well as facilitating the unpacking of DNA from the complexes. GALA was also added to lipoplexes formed by the interaction of DNA with cationic liposomes composed of DOTAP. Futaki et al. showed that mixing GALA with cationic lipoplexes improved transfection activities, especially when Lipofectin is used ${ }^{22}$. Surprisingly, the presence of GALA reduced the cellular uptake and only slightly improved endosomal escape, indicating that even a slight improvement in endosomal escape is reflected as a significant improvement in gene expression ${ }^{22}$. It is also possible that GALA may improve the nuclear delivery of DNA and/or improve the efficiency of transcription/translation processes. The next step involved the chemical conjugation of GALA with different cationic polymers or lipids. GALA, when conjugated to a cationic dendrimer, enhanced gene expression through improved endosomal escape ${ }^{15}$. As explained earlier, the positive variant, KALA, can spontaneously bind to DNA and condense it into stable NPs with a high transfection activity ${ }^{17}$. KALA can enhance cellular uptake as well as endosomal escape. Furthermore, KALA has been successfully used for improving the nuclear delivery of plasmid DNA (pDNA) in non-dividing cells ${ }^{23}$.

For applying GALA to gene delivery, we prepared Chol-GALA to attach the peptide to the surface of LNPs. Kakudo et al. prepared Tf-modified liposomes (Tf-Lip) encapsulating a rhodamine probe as a model hydrophilic drug and equipped it with Chol-GALA for improving intracellular trafficking ${ }^{16}$. The incorporation of Chol-GALA efficiently released the encapsulated rhodamine in the cytosol in a pH-dependent manner. This release is correlated with lipid mixing and fusion between liposomes and the endosomal membrane. The addition of GALA to a Tf-Lip suspension or the encapsulation of GALA in Tf-Lip did not cause rhodamine release ${ }^{16}$. These results indicated that using a cholesterol moiety attached to GALA is essential for correctly exposing the peptide on the surface of liposomes to appropriately interact with the endosomal membrane. Sasaki et al. encapsulated pDNA in Tf-Lip and used CholGALA or GALA attached to polyethylene glycol (PEG) to control intracellular trafficking $^{24}$. Chol-GALA and PEG-GALA interacted synergistically to induce the efficient endosomal escape of pDNA and transfection activity was increased by two orders of magnitude. Yamada et al. prepared Tf-Lip encapsulating mastoparan, an antitumor agent which facilitates mitochondrial permeability 
$\operatorname{transition}^{25}$. In the presence of Chol-GALA, mastoparan was efficiently delivered to mitochondria and cytochrome $\mathrm{c}$ was released to the cytosol. This effect was not observed when mastoparan was delivered by means of a Tf-Lip lacking Chol-GALA ${ }^{25}$.

Our group developed a multi-functional envelope-type nanodevice (MEND) for efficient gene delivery in-vitro and in$v_{i v o} 2,26 \& 27$. The presence of Chol-GALA in the lipid surface of the MEND significantly improved the activity. Sakurai et al. prepared a MEND encapsulating short interfering RNA (siRNA) and modified it with an octaarginine (R8) peptide $^{28}$. Efficient gene silencing was observed in HeLa cells stably expressing luciferase (HeLa-Luc), but only when CholGALA was incorporated in the MEND structure. Silencing activity was further improved by using a cleavable tumor specific PEG-lipid ${ }^{29 \& 30}$. Akita et al. used an R8-MEND modified with Chol-GALA for ex-vivo transfecting primary bone marrow-derived dendritic cells (BMDCs) with siRNA against suppressor of cytokine signaling 1 (SOCS1) and transfected cells were successfully used an anti-rumor vaccine ${ }^{30}$. Collectively, these results confirm the value of Chol-GALA in enhancing the endosomal escape and gene delivery efficiency in different systems.

\section{2- In-vivo gene delivery}

The MEND system was also successfully used for in-vivo gene delivery and the activity was dramatically enhanced in the presence of Chol-GALA. The different versions of GALAMEND that were used for in-vivo targeted gene delivery are shown in figure 2. Hatakeyama $e t$ al. used a GALA-MEND for gene silencing after the topical administration of siRNA ${ }^{29}$. MEND modified with a cleavable-PEG derivative could be used for siRNA delivery to tumor tissues after systemic administration ${ }^{31}$. Yamauchi et al. compared the transfection activity of a MEND with Lipotrust, a commercially available transfection reagent, after intravenous (IV) administration ${ }^{32}$. Lipotrust transfected the lung at a 5 fold higher level than the liver, which reflected the aggregation of cationic particles and entrapment in the lung capillaries. In contrast, the MEND transfected the liver more efficiently and the activity was substantially improved when Chol-GALA was introduced. Chol-GALA improved the activity by 18 times in liver parenchymal cells and by 11 times in liver non-parenchymal cells $\mathrm{s}^{32}$. This indicates that GALA is an efficient tool for improving gene delivery to different types of liver cells.

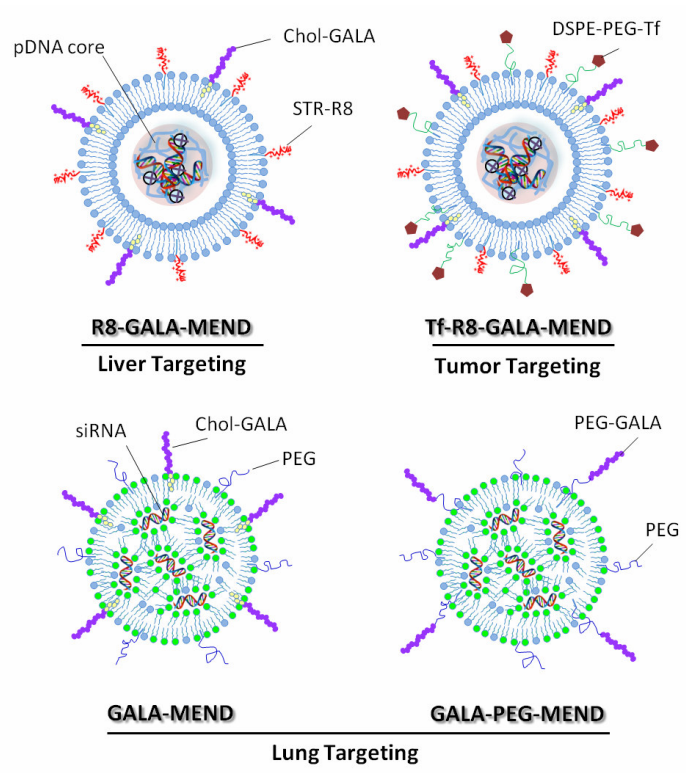

Fig. 2: A schematic representation of the different versions of GALA-modified MENDs applied for in-vivo gene delivery. The R8-GALAMEND targets liver hepatocytes due to the affinity of hepatocytes for R8. The Tf-R8GALA-MEND is used for tumor targeting. PEG imparts a long circulation and tarnsferrin (Tf) is used to enhance cellular uptake in tumor cells. The GALA-MEND is used for the targeted delivery of siRNA to the lung endothelium. In the GALA-PEGMEND, adding a PEG-spacer between the GALA and the MEND surface further improved gene delivery.

Liposomes modified with R8 (R8-Lip) encapsulating pDNA showed a high transfection activity in-vitro but failed to produce a similarly high activity in-vivo ${ }^{33}$. In studying the biodistribution of R8-Lip after IV administration, we found that most of the dose is delivered to the liver ${ }^{34}$. The cationic nature of the system probably caused extensive binding to negatively charged serum proteins and the opsonized NPs were mainly taken up by liver macrophages and then degraded. Therefore, we speculated that the addition of GALA would improve transfection activity in 
the liver. R8-MEND prepared with positively charged DNA core covered with a lipid envelope failed to produce gene expression even in the presence of Chol-GALA ${ }^{35}$. However, a high level of gene expression was observed in the liver when an R8-MEND containing Chol-GALA was prepared using a negatively charged DNA core. We hypothesized that DNA decondensation is facilitated when DNA is used as a negative core. However, measurement of the pDNA delivered to the nucleus showed that a slightly higher amount of DNA was delivered to the nucleus in the presence of GALA, irrespective of whether the MEND was prepared with positive or negative cores ${ }^{35}$. This suggests that GALA function might exceed the endosomal escape process and might affect post-nuclear events. Our data raised the possibility that GALA may affect transcription and/or translation processes. Although the role of GALA in post-nuclear events is ambiguous and the mechanism of this suggested effect is not clear, the activity of the optimized R8-GALAMEND in the liver was dramatically higher than that for the R8-MEND without GALA $(\sim 6000 \text { fold })^{35}$. Furthermore, the activity was substantially lower in other organs such as the lung and spleen, indicating that this system can be used for targeted delivery to the liver, although this study did not examine the type of liver cells transfected. The activity of the optimized R8-GALA-MEND in the liver was higher than that for a GALA-MEND prepared with the cationic lipid DOTAP ${ }^{35}$. Collectively, these results indicate that the R8-GALAMEND is a promising system for liver selective gene delivery. This system was also successfully used for the delivery of siRNA to the liver ${ }^{36}$. A MEND encapsulating siRNA against a scavenger receptor class B, member 1 (SR-BI) caused efficient gene silencing in liver cells after IV administration without adverse effects. Since this gene is located in liver hepatocytes, this result confirms that the R8GALA-MEND has the ability to transfect hepatocytes. We further used an R8-GALAMEND for protecting liver hepatocytes from toxicity induced by lipopolysaccharide/Dgalactosamine (LPS/D-GalN) ${ }^{37}$. Plasmid DNA encoding the hepatocyte growth factor (HGF) was encapsulated into the R8-GALA-MEND and injected into mice prior to the administration of LPS/D-GalN. Measurement of indicators of acute liver injury including alanine aminotransferase (ALT) and aspartate aminotransferase (AST) indicated that R8GALA-MEND encapsulating pDNA encoding HGF efficiently protected the liver against acute toxicity caused by LPS/D-GalN. The protection was significantly higher than that caused by jet-PEI-Gal, a commercially available liver-specific transfection reagent ${ }^{37}$. The GALA peptide can thus be used for gene delivery both in-vitro and in-vivo.

\section{The role of GALA in gene delivery: Lung endothelium targeting 1- Binding to lung endothelial cells}

Although GALA is commonly used for its ability to induce membrane fusion and improving the endosomal escape of endocytosed materials, our group accidentally discovered that GALA has a unique ability to bind to lung endothelial cells $\left(\right.$ LECs) ${ }^{10}$. Kusumoto et al. reported that liposomes modified with GALA (GALA-Lip) are selectively delivered to the lung endothelium after IV administration ${ }^{10}$. Normally, cationic liposomes bind to negative serum proteins and form aggregates, which accumulate in the lung capillaries since they are the first capillaries that are encountered after IV administration. This was not the case with the GALA-Lip, which has a net negative charge. The GALALip was delivered to LECs as single particles without aggregation as judged by visualizing the dynamic flow of GALA-Lip in live mice using intravital real time confocal microscopy (IVRTCLSM). GALA-Lip rapidly accumulate in the lung within $5 \mathrm{~min}$ after injection and remained there for at least $6 \mathrm{hr}$, which is different from cationic liposomes that accumulate in the lung after 5 min but eventually cleared from the lung after $1 \mathrm{hr}^{10}$. GALA-Lip was preferentially taken up by endothelial-derived cell lines compared to other cell lines and the uptake was blocked by the presence of excess free GALA, indicating that a specific uptake mechanism is involved. The uptake of GALA-Lip was blocked in the presence of different lectins, which block the binding to sugar chains terminated with sialic acid residues ${ }^{10}$. This indicated that the uptake of GALA-Lip into endothelial cells is similar to the uptake of hemagglutinin 2 (HA2) subunit of the influenza virus. 


\section{2- Application in siRNA delivery to the lung endothelium}

Inspired by the finding that the GALA-Lip preferentially accumulates in the lung endothelium after IV administration, Kusumoto et al. prepared a GALA-MEND encapsulating siRNA against CD31 gene and used it for specific gene silencing in the lung endothelium ${ }^{10}$. Gene delivery to the lung endothelium is a promising approach for treating a variety of lung diseases. Using IVRTCLSM, the authors observed the flow of a GALA-MEND in the lung capillaries and found that the particles accumulated along the blood vessels of the lung without aggregation. A strong gene silencing $(\sim 90 \%)$ could be observed in the lung using a dose of $1 \mathrm{mg}$ siRNA/kg, which was specific for the lung endothelium (the $50 \%$ effective dose concentration value (ED50) was $\sim 0.4 \mathrm{mg}$ siRNA $/ \mathrm{kg})^{10}$. Because the CD31 gene is involved in the progression of metastatic melanoma, GALA-MEND prevented the progression of pulmonary metastasis of melanoma cells to $\sim 50 \%$ compared to untreated group $^{10}$. The GALA peptide here played a dual function; targeting the lung endothelium and enhancing the endosomal escape.

The GALA-MEND described in the previous study was prepared by a lipid film hydration method. The ethanol dilution method is an alternative method for preparing GALAMEND, which is more preferable for particle homogeneity and scale-up. A GALA-MEND prepared by ethanol dilution was compared to those prepared by film hydration ${ }^{38}$. Both MENDs accumulated in the lung to the same extent, however, the MEND prepared by ethanol dilution showed a stronger gene silencing in the lung. A mechanistic comparison showed that the endosomal escape ability of the GALA-MEND was improved when prepared using the ethanol dilution method $^{38}$. Santiwarangkool et al. further improved the lung accumulation of the GALAMEND by using Chol-PEG-GALA, in which a PEG spacer is added between GALA and cholesterol $^{39}$. The PEG spacer improved the flexibility of the GALA peptide and improved the interaction with sugars located on the surface of endothelial cells. The gene silencing of the GALA-MEND was improved by using ssPalmE, a vitamin E-scaffold SS-cleavable
$\mathrm{pH}$-activated lipid-like material (ED50 was $\sim 0.2 \mathrm{mg}$ siRNA $/ \mathrm{Kg})^{39}$.

The efficiency of gene silencing using siRNA encapsulated in LNPs was dramatically increased by the introduction of $\mathrm{pH}$-sensitive cationic lipids $^{40 \& 41}$. However, these lipids have an inherent ability to target liver hepatocytes after binding to specific endogenous proteins ${ }^{42}$. Therefore, it was a challenging task to incorporate a $\mathrm{pH}$-sensitive cationic lipid in the GALA-MEND system for improving the efficiency of gene silencing in the lung without affecting its lung selectivity. We incorporated YSK05, a pH-sensitive cationic lipid that was originally synthesized in our laboratory ${ }^{40 \& 43}$, in the case of the GALA-MEND, hoping to improve gene silencing efficiency in the lung. The GALA/YSK05-MEND showed improved gene silencing in the lung, however, considerable gene silencing was also observed in the liver, indicating that lung selectivity was largely $\operatorname{lost}^{44}$. In an attempt to improve lung selectivity, we changed the lipid composition by using different types of helper lipids. Surprisingly, using DOPE, a fusogenic lipid that is commonly used as a helper lipid, significantly reduced the accumulation and gene silencing in the liver ${ }^{44}$. Furthermore, the presence of DOPE improved gene silencing in LECs in-vitro and in-vivo. The activity of the optimized GALA-MEND was improved by $\sim 40$ fold compared to the original MEND prepared by Kusumoto et al. (ED50 was $\sim 0.01 \mathrm{mg}$ siRNA/Kg $)^{10 \& 44}$. Meanwhile, using a dose 50 times higher than the ED50, only a slight gene silencing was observed in the liver, while no significant gene silencing was observed in other organs. Flow cytometry analysis confirmed that the siRNA was delivered to $>90 \%$ of LECs. The GALA/YSK05-MEND was successfully used for preventing the progression of lung metastasis without detectable adverse effects ${ }^{44}$. The acute and chronic toxicity of the GALA/YSK05-MEND was examined in healthy mice and in mice with lung metastasis. In the acute toxicity evaluation, the siRNA dose equivalent to 50 times the ED50 was injected into healthy mice and different biochemical markers of toxicity were measured in the serum $24 \mathrm{hr}$ later. There was no significant difference in biochemical markers for toxicity between the treated and untreated mice, indicating that the GALA- 
MEND is a well-tolerated system. In the chronic toxicity evaluation, mice with metastatic lung cancer were treated 6 times with an siRNA dose equivalent to 50 times the ED50 and toxicity markers were evaluated 1 day after the last injection. The levels of toxicity markers were comparable to those for untreated mice except for markers of liver toxicity, which were decreased, indicating that the inhibition of lung metastasis further inhibited liver metastasis. Collectively, these results confirm that GALA is a valuable tool for the targeted gene delivery to the lung and highlights the importance of modulating the lipid content to improve the targeting function.

\section{3- Application in pDNA delivery to the lung endothelium}

After being successfully used for siRNA delivery to the lung, we attempted to use the GALA-MEND for the delivery of pDNA. The delivery of pDNA is more challenging compared to siRNA because it has a higher molecular weight and contains a higher negative charge ${ }^{45}$. In addition, pDNA must be delivered to the nucleus, unlike siRNA which functions in the cytosol. We prepared a GALAMEND encapsulating pDNA and examined the gene expression efficiency in different organs. The transfection activity in the lung was comparable to that in the liver and spleen. However, a biodistribution study indicated that the pDNA is delivered to the lung more than to other organs. Therefore, the low efficiency in the lung can be attributed to poor intracellular trafficking rather than poor delivery to lung cells. To improve the gene expression efficiency in the lung, we prepared a GALAMEND containing the cationic lipid, DOTAP and the R8 peptide. The presence of DOTAP improved encapsulation efficiency of pDNA and the presence of R8 improved the nuclear delivery $^{46}$. The modified GALA-MEND produced a high gene expression in lung endothelial cells in-vitro (unpublished data). This indicates that GALA can be used for pDNA as well as siRNA delivery to the lung.

\section{4- Transcytosis}

As described earlier, GALA-Lip was efficiently delivered to the lung endothelium. However, we also observed that a fraction of the GALA-Lip could pass through the endothelial barrier, ultimately reaching alveolar epithelial cells and alveolar macrophages ${ }^{47}$. Using electron microscopy and flow cytometry, we found that GALA-Lip was delivered to $\sim 30 \%$ of type 1 alveolar epithelial cells (ATI). Since the endothelium in the lung capillaries is continuous and shows no large fenestrations, it is unlikely that GALA-Lip (with diameter $\sim 100$ $\mathrm{nm}$ ) would pass through the paracellular route ${ }^{4}$. We proposed that the GALA-Lip undergoes transcytosis in LECs to reach other pulmonary cells. This was confirmed by observing a strong gene silencing of podoplanin, a gene located in alveolar epithelial cells, when GALA-MEND encapsulated the anti-podoplanin siRNA ${ }^{47}$. This indicates that GALA is not only a targeting ligand to the lung endothelium, but can also be used to deliver macromolecules to other pulmonary cells.

\section{Synergism with cell penetrating peptides}

For tumor targeting, the LNPs are commonly coated with a hydrophilic polymer such as PEG to extend the circulation in the blood after IV administration. This long circulation allows NPs to passively target the tumor tissues through the enhanced permeability and retention (EPR) effect ${ }^{48}$. The tips of the PEG chains are sometimes modified with specific ligands that recognize certain molecules on the surface of tumor cells to improve cellular uptake through active targeting ${ }^{4 \& 49}$. However, the presence of a PEG coating is a strong inhibitor of endosomal escape since it represents a barrier that prevents the interaction between the lipid membranes of the carrier and endosomes ${ }^{11}$. In this instance, GALA appears to be a valuable device for improving endosomal escape in the presence of PEG. We previously showed that a Tf-PEGMEND encapsulating pDNA can be used for actively targeting tumor cells ${ }^{11}$. However, the transfection activity was very low, despite the high cellular uptake mediated by $\mathrm{Tf}$ receptors that are overexpressed in tumor cells. The inclusion of Chol-GALA improved the activity by $\sim 1$ fold, which was not sufficient for eliciting a therapeutic anti-tumor effect. We incorporated the $\mathrm{R} 8$ peptide into the system and examined the transfection activities in the presence or absence of GALA. Surprisingly, the use of a combination of R8 and GALA improved the activities by $\sim 400$ fold, compared 
to the system prepared with R8 or GALA alone $^{11}$. In this design, the PEG amount is adjusted to completely shield the positive charge of R8 to avoid non-specific interactions with serum components and to confirm the long circulation properties required for tumor targeting. Also, the Tf ligands are localized on the tip of PEG chains thus permitting them to have sufficient flexibility to interact with Tf receptors on tumor cells. The addition of excess free Tf strongly suppressed gene expression, which confirmed that the system was internalized into tumor cells through $\mathrm{Tf}$ receptors. The use of octalysine (K8), a positively charged peptide similar to R8, failed to produce the same synergistic combination with GALA. This shows that the R8 effect is not due exclusively to its positive charge only, but it is mainly related to its chemical structure. The Tf-MEND modified with R8 and GALA produced a gene expression comparable to the Lipofectamine Plus reagent, one of the strongest transfection reagents, in HeLa cells even in the presence of serum, without substantial cell toxicity as judged by the total protein content in cells ${ }^{11}$. In the absence of R8, Tf ligands are separated from $\mathrm{Tf}$ receptors in the acidic $\mathrm{pH}$ of endosomes and the PEG coat inhibits the interaction between GALA and the endosomal membrane. In contrast, the presence of R8 improves the binding of the lipids of the carrier to the cellular membranes and this facilitates the interaction between GALA and endosomal membrane. This triple-ligand system (Tf, R8 and GALA) is highly promising for future applications in tumor targeting.

\section{Uptake mechanism of GALA-modified nanoparticles}

To understand the interaction between GALA and LECs, we investigated the cellular uptake mechanism of GALA-Lip in human lung microvascular endothelial cells (HMVEC$\mathrm{L})^{47}$. The uptake and subsequent transcytosis in endothelial cells is closely linked to the uptake through caveolae, which are present in abundance on the surface of endothelial cells ${ }^{8}$. However, the uptake of GALA-Lip into endothelial cells was only slightly inhibited in the presence of filipin III, an inhibitor of uptake through caveolae ${ }^{47}$. Although the HA2 protein of the influenza virus, which mimics GALA, is taken up by macropinocytosis in different cell lines $^{50}$, the uptake of the GALA-Lip was only slightly inhibited in the presence of amiloride, an inhibitor of macropinocytosis ${ }^{51}$. The uptake of GALA-Lip was strongly inhibited in the presence of chloropromazine and this inhibition was dose-dependent, indicating that clathrin-mediated endocytosis is the major uptake pathway for GALA-modified systems, at least in lung endothelial cells ${ }^{47}$. These results were confirmed by using confocal microscopy, which confirmed that the GALA-Lip was more colocalized with $\mathrm{Tf}$, which are internalized by clathrin-mediated endocytosis and were less colocalized with Cholera Toxin Subunit B (CTB) or dextran, which are internalized by caveolae and macropinocytosis, respectively. Furthermore, the activity of the GALA-MEND encapsulating anti CD31 siRNA was significantly inhibited in the presence of chloropromazine. Collectively, these results suggest that clathrin-mediated endocytosis represents a major uptake pathway in lung endothelial cells.

\section{Conclusion}

The GALA peptide discussed in this review is a promising tool for gene delivery. It plays multiple roles including targeting to the lung endothelium and improving the endosomal escape of drugs. Furthermore, it acts synergistically with certain cell penetrating peptides to improve gene expression. The MEND system modified with GALA proved to be an efficient platform for the in-vitro and invivo delivery of different nucleic acids including pDNA, siRNA and mRNA. Understanding the nature of GALA and its properties will permit further optimization for developing more efficient non-viral gene vectors. In addition, further work will be needed to apply different GALA-modified systems for clinical use.

\section{Conflict of interest (COI)}

"The authors declare no conflict of interest."

\section{REFERENCES}

1- M. Collins and A. Thrasher, "Gene therapy: Progress and predictions", Proc. Biol. Sci., 282, 20143003 (2015). 
2- K. Kogure, H. Akita, Y. Yamada and H. Harashima, "Multifunctional envelopetype nano device (MEND) as a non-viral gene delivery system", Adv. Drug Deliv. Rev., 60, 559-571 (2008).

3- A. N. Lukashev and A. A. Zamyatnin, "Viral vectors for gene therapy: Current state and clinical perspectives", Biochemistry (Mosc), 81, 700-708 (2016).

4- I. A. Khalil, Y. Sato and H. Harashima, "Recent advances in the targeting of systemically administered non-viral gene delivery systems", Expert. Opin. Drug Deliv., 16, 1-14 (2019).

5- H. Yin, R. L. Kanasty, A. A. Eltoukhy, A. J. Vegas, J. R. Dorkin and D. G. Anderson, "Non-viral vectors for genebased therapy", Nat. Rev. Genet., 15, 541555 (2014).

6- T. Nakamura, Y. Yamada, Y. Sato, I. A. Khalil and H. Harashima, "Innovative nanotechnologies for enhancing nucleic acids/gene therapy: Controlling intracellular trafficking to targeted biodistribution", Biomaterials, 218, 119329 (2019).

7- F. Alexis, E. Pridgen, L. K. Molnar and O. C. Farokhzad, "Factors affecting the clearance and biodistribution of polymeric nanoparticles", Mol. Pharm., 5, 505-515 (2008).

8- I. A. Khalil, K. Kogure, H. Akita and H. Harashima, "Uptake pathways and subsequent intracellular trafficking in nonviral gene delivery", Pharmacol. Rev., 58, 32-45 (2006).

9- W. Li, F. Nicol and F. C. Szoka Jr., "GALA: A designed synthetic pHresponsive amphipathic peptide with applications in drug and gene delivery", Advanced Drug Delivery Reviews, 56, 967-985 (2004).

10- K. Kusumoto, H. Akita, T. Ishitsuka, Y. Matsumoto, T. Nomoto, R. Furukawa, et al., "Lipid envelope-type nanoparticle incorporating a multifunctional peptide for systemic siRNA delivery to the pulmonary endothelium", ACS Nano, 7, 7534-7541 (2013).

11- I. A. Khalil and H. Harashima, "An efficient PEGylated gene delivery system with improved targeting: Synergism between octaarginine and a fusogenic peptide", Int. J. Pharm., 538, 179-187 (2018).

12- N. K. Subbarao, R. A. Parente, F. C. Szoka Jr., L. Nadasdi and K. Pongracz, "pH-dependent bilayer destabilization by an amphipathic peptide", Biochemistry, 26, 2964-2972 (1987).

13- C. Plank, B. Oberhauser, K. Mechtler, C. Koch and E. Wagner, "The influence of endosome-disruptive peptides on gene transfer using synthetic virus-like gene transfer systems", The Journal of Biological Chemistry, 269, 12918-12924 (1994).

14- S. Simões, V. Slepushkin, E. Pretzer, P. Dazin, R. Gaspar, M. C. Pedroso de Lima, et al., "Transfection of human macrophages by lipoplexes via the combined use of transferrin and $\mathrm{pH}$ sensitive peptides", Journal of Leukocyte Biology, 65, 270-279 (1999).

15- J. Haensler and F. C. Szoka Jr., "Polyamidoamine cascade polymers mediate efficient transfection of cells in culture", Bioconjugate Chemistry, 4, 372379 (1993).

16- T. Kakudo, S. Chaki, S. Futaki, I. Nakase, K. Akaji, T. Kawakami, et al., "Transferrin-modified liposomes equipped with a $\mathrm{pH}$-sensitive fusogenic peptide: An artificial viral-like delivery system", Biochemistry, 43, 5618-5628 (2004).

17- T. B. Wyman, F. Nicol, O. Zelphati, P. V. Scaria, C. Plank and F. C. Szoka Jr., "Design, synthesis, and characterization of a cationic peptide that binds to nucleic acids and permeabilizes bilayers", ibid., 36, 3008-3017 (1997).

18- E. Goormaghtigh, J. De Meutter, F. Szoka, V. Cabiaux, R. A. Parente and J. M. Ruysschaert, "Secondary structure and orientation of the amphipathic peptide GALA in lipid structures. An infraredspectroscopic approach", European Journal of Biochemistry, 195, 421-429 (1991).

19- R. A. Parente, L. Nadasdi, N. K. Subbarao and F. C. Szoka Jr., "Association of a pHsensitive peptide with membrane vesicles: Role of amino acid sequence". Biochemistry, 29, 8713-8719 (1990).

20- R. A. Parente, S. Nir and F. C. Szoka Jr., "pH-dependent fusion of phosphatidyl- 
choline small vesicles. Induction by a synthetic amphipathic peptide", The Journal of Biological Chemistry, 263, 4724-4730 (1988).

21- R. A. Parente, S. Nir and F. C. Szoka Jr., "Mechanism of leakage of phospholipid vesicle contents induced by the peptide GALA", Biochemistry, 29, 8720-8728 (1990).

22- S. Futaki, Y. Masui, I. Nakase, Y. Sugiura, T. Nakamura, K. Kogure, et al., "Unique features of a pH-sensitive fusogenic peptide that improves the transfection efficiency of cationic liposomes", The Journal of Gene Medicine, 7, 1450-1458 (2005).

23- S. M. Shaheen, H. Akita, T. Nakamura, S. Takayama, S. Futaki, A. Yamashita, et al., "KALA-modified multi-layered nanoparticles as gene carriers for MHC class-I mediated antigen presentation for a DNA vaccine", Biomaterials, 32, 6342-6350 (2011).

24- K. Sasaki, K. Kogure, S. Chaki, Y. Nakamura, R. Moriguchi, H. Hamada, et al., "An artificial virus-like nano carrier system: Enhanced endosomal escape of nanoparticles via synergistic action of $\mathrm{pH}$ sensitive fusogenic peptide derivatives", Analytical and Bioanalytical Chemistry, 391, 2717-2727 (2008).

25- Y. Yamada, Y. Shinohara, T. Kakudo, S. Chaki, S. Futaki, H. Kamiya, et al., "Mitochondrial delivery of mastoparan with transferrin liposomes equipped with a $\mathrm{pH}$-sensitive fusogenic peptide for selective cancer therapy", International Journal of Pharmaceutics, 303, 1-7 (2005).

26- I. A. Khalil, K. Kogure, S. Futaki, S. Hama, H. Akita, M. Ueno, et al., "Octaarginine-modified multifunctional envelope-type nanoparticles for gene delivery", Gene Ther., 14, 682-689 (2007).

27- $\mathrm{H}$. Hatakeyama, H. Akita and $\mathrm{H}$. Harashima, "A multifunctional envelope type nano device (MEND) for gene delivery to tumours based on the EPR effect: A strategy for overcoming the PEG dilemma", Adv. Drug Deliv. Rev., 63, 152160 (2011).
28- Y. Sakurai, H. Hatakeyama, H. Akita, M. Oishi, Y. Nagasaki, S. Futaki, et al., "Efficient short interference RNA delivery to tumor cells using a combination of octaarginine, GALA and tumor-specific, cleavable polyethylene glycol system", Biological \& Pharmaceutical Bulletin, 32, 928-932 (2009).

29- H. Hatakeyama, E. Ito, H. Akita, M. Oishi, Y. Nagasaki, S. Futaki, et al., "A pHsensitive fusogenic peptide facilitates endosomal escape and greatly enhances the gene silencing of siRNA-containing nanoparticles in-vitro and in-vivo", Journal of Controlled Release: Official Journal of the Controlled Release Society, 139, 127-132 (2009).

30- H. Akita, K. Kogure, R. Moriguchi, Y. Nakamura, T. Higashi, T. Nakamura, et al., "Nanoparticles for ex-vivo siRNA delivery to dendritic cells for cancer vaccines: Programmed endosomal escape and dissociation", ibid., 143, 311-317 (2010).

31- H. Hatakeyama, H. Akita, K. Kogure and H. Harashima, "A novel nonviral gene delivery system: Multifunctional envelope-type nano device". $\boldsymbol{A d \boldsymbol { d }}$. Biochem. Eng. Biotechnol., 119, 197-230 (2010).

32- J. Yamauchi, Y. Hayashi, K. Kajimoto, H. Akita and H. Harashima, "Comparison between a multifunctional envelope-type nano device and lipoplex for delivery to the liver", Biological \& Pharmaceutical Bulletin, 33, 926-929 (2010).

33- I. A. Khalil, K. Kogure, S. Futaki and H. Harashima, "Octaarginine-modified liposomes: Enhanced cellular uptake and controlled intracellular trafficking", Int. J. Pharm., 354, 39-48 (2008).

34- D. Mudhakir, H. Akita, I. A. Khalil, S. Futaki and H. Harashima, "Pharmacokinetic analysis of the tissue distribution of octaarginine modified liposomes in mice", Drug Metab. Pharmacokinet., 20, 275-281 (2005).

35- I. A. Khalil, Y. Hayashi, R. Mizuno and H. Harashima, "Octaarginine- and $\mathrm{pH}$ sensitive fusogenic peptide-modified nanoparticles for liver gene delivery", $\boldsymbol{J}$. Control. Release, 156, 374-380 (2011). 
36- Y. Hayashi, J. Yamauchi, I. A. Khalil, K Kajimoto, H. Akita and H. Harashima, "Cell penetrating peptide-mediated systemic siRNA delivery to the liver", International Journal of Pharmaceutics, 419, 308-313 (2011).

37- Y. Hayashi, R. Mizuno, K. A. Ikramy, H. Akita and H. Harashima, "Pretreatment of hepatocyte growth factor gene transfer mediated by octaarginine peptidemodified nanoparticles ameliorates LPS/D-galactosamine-induced hepatitis", Nucleic Acid Ther., 22, 360-363 (2012).

38- K. Kusumoto, H. Akita, S. Santiwarangkool and H. Harashima, "Advantages of ethanol dilution method for preparing GALA-modified liposomal siRNA carriers on the in-vivo gene knockdown efficiency in pulmonary endothelium", Int. J. Pharm., 473, 144 147 (2014).

39- S. Santiwarangkool, H. Akita, T. Nakatani, K. Kusumoto, H. Kimura, M. Suzuki, et al., "PEGylation of the GALA Peptide Enhances the Lung-Targeting Activity of Nanocarriers That Contain Encapsulated siRNA", J. Pharm. Sci., 106, 2420-2427 (2017).

40- Y. Sato, H. Hatakeyama, Y. Sakurai, M. Hyodo, H. Akita and H. Harashima, "A pH-sensitive cationic lipid facilitates the delivery of liposomal siRNA and gene silencing activity in-vitro and in-vivo", $\boldsymbol{J}$. Control. Release, 163, 267-276 (2012).

41- D. Adams, A. Gonzalez-Duarte, W. D. O'Riordan, C. C. Yang, M. Ueda, A. V. Kristen, et al., "Patisiran, an RNAi Therapeutic, for Hereditary Transthyretin Amyloidosis", N. Engl. J. Med., 379, 1121 (2018).

42- J. Yang, "Patisiran for the treatment of hereditary transthyretin-mediated amyloidosis", Expert. Review of Clinical Pharmacology, 12, 95-99 (2019).

43- I. A. Khalil, S. Kimura, Y. Sato and H. Harashima, "Synergism between a cell penetrating peptide and a $\mathrm{pH}$-sensitive cationic lipid in efficient gene delivery based on double-coated nanoparticles", $\boldsymbol{J}$. Control. Release, 275, 107-116 (2018).

44- M. M. Abd Elwakil, I. A. Khalil, Y. H. A. Elewa, K. Kusumoto, Y. Sato, N. Shobaki, et al., "Lung-endothelium-targeted nano- particles based on a $\mathrm{pH}$-sensitive lipid and the GALA peptide enable robust gene silencing and the regression of metastatic lung cancer", Advanced Functional Materials, 29, 1807677 (2019).

45- I. A. Khalil, Y. Yamada and H. Harashima, "Optimization of siRNA delivery to target sites: Issues and future directions", Expert. Opin. Drug Deliv., 113 (2018)

46- S. Kimura, I. A. Khalil, Y. H. A. Elewa and H. Harashima, "Spleen selective enhancement of transfection activities of plasmid DNA driven by octaarginine and an ionizable lipid and its implications for cancer immunization", Journal of Controlled Release: Official Journal of the Controlled Release Society, 313, 7079 (2019).

47- S. Santiwarangkool, H. Akita, I. A. Khalil, M. M. Abd Elwakil, Y. Sato, K. Kusumoto, et al., "A study of the endocytosis mechanism and transendothelial activity of lung-targeted GALAmodified liposomes", J. Control. Release, 307, 55-63 (2019).

48- H. Maeda, J. Wu, T. Sawa, Y. Matsumura and K. Hori, "Tumor vascular permeability and the EPR effect in macromolecular therapeutics: A review", ibid., 65, 271-284 (2000).

49- M. A. Younis, I. A. Khalil, M. M. Abd Elwakil and H. Harashima, "A multifunctional lipid-based nanodevice for the highly specific codelivery of sorafenib and midkine siRNA to hepatic cancer cells", Molecular Pharmaceutics, 16, 4031-4044 (2019).

50- S. Kannan, R. Shankar and P. Kolandaivel, "Insights into structural and inhibitory mechanisms of low $\mathrm{pH}$-induced conformational change of influenza HA2 protein: A computational approach", Journal of Molecular Modeling, 25, 9999 (2019).

51- I. A. Khalil, K. Kogure, S. Futaki and H. Harashima, "High density of octaarginine stimulates macropinocytosis leading to efficient intracellular trafficking for gene expression", J. Biol. Chem., 281, 35443551 (2006). 


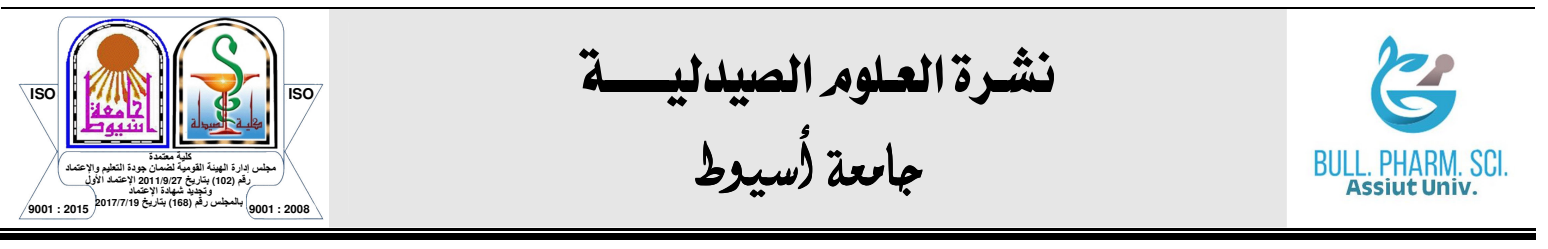

\section{بيتيد الجالا: أدوار متعددة في ثوصيل الأدوية والجينات

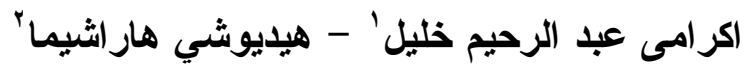

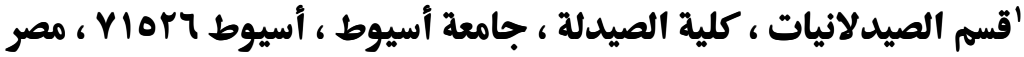

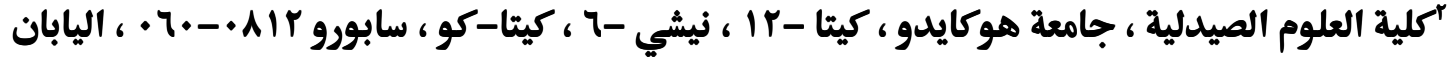

الأحماض النووية العلاجية يجب أن تصاغ في أنظمة توصبل مناسبة توصلها بكفاءة إلى أنسجة

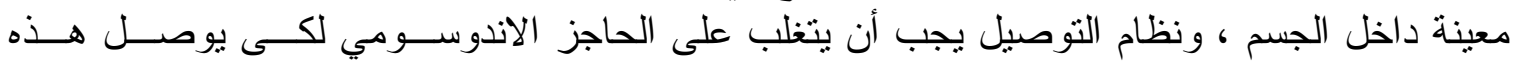

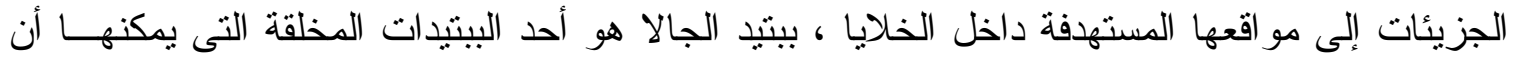

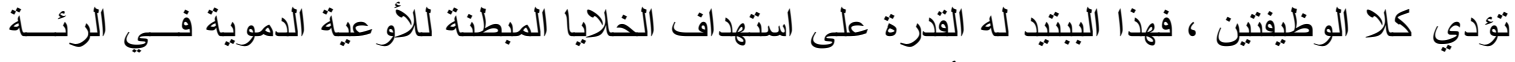

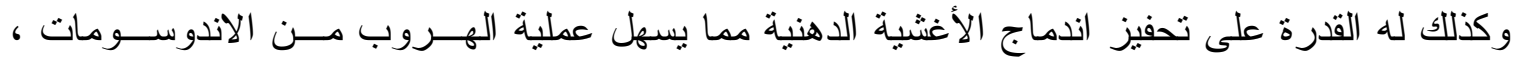

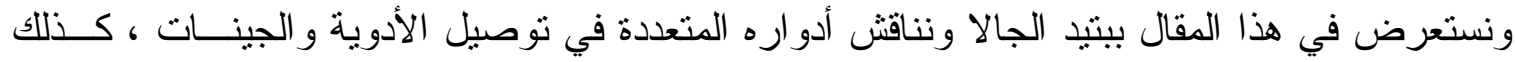

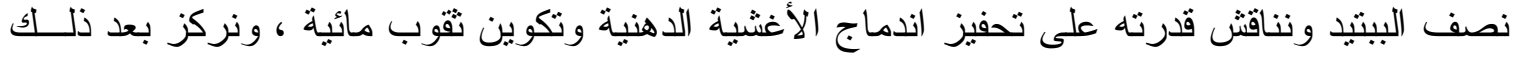

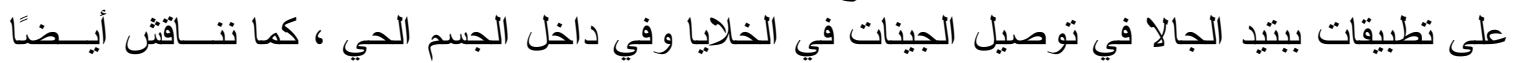

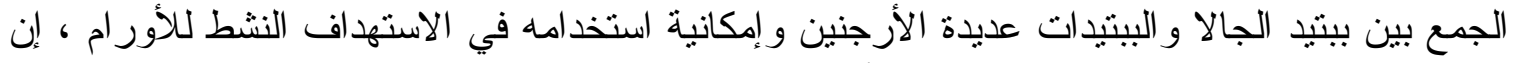
ببتيد الجالا الذي نقدمه في هذا المقال يعتبر أداة و اعدة لتوصيل الجينات بكفاءة. 